\title{
ON THE CALCULATION OF LOW FREQUENCY OSCILLATIONS OF THE EARTH'S CORE
}

\author{
S.V. DYAKONOV \\ O.Yu. Shmidt Institute of Earth Physics \\ Bolshaya Gruzinskaya 10 \\ 123810 Moscow D-242 \\ USSR
}

ABSTRACT. The problem of calculation of low frequency oscillations of an ideal rotating compressible fluid is investigated. An original method of solving such a problem, based on using characteristic functions of the Poincaré operator is proposed. An efficient scheme of calculating the characteristic numbers and functions of the Poincaré operator is worked out. The high speed of convergence of the method is shown. An essential influence of compressibility on the theoretical nutation amplitude is found.

While calculating low frequency oscillations of the Earth's fluid core (e.g., for calculation of the forced nutation amplitude), spherical harmonic representation of the deformation field is usually used [1-3]:

$$
u=\sum_{l} \sum_{m}\left[S_{l}^{m}+T_{l}^{m}\right]
$$

Substitution of Eq. (1) into the equations of motion gives an infinite system of differential equations for the scalar functions $S_{l}^{m}$ and $T_{l}^{m}$. Approximate solutions of such a system are obtained by truncating the system of equations. Probably one cannot prove the convergence of the set of such approximate solutions. The author has proposed an alternative method for calculation of low frequency oscillations of a fluid Earth core.

Let us calculate the forced nutation amplitude for the Earth model consisting of an absolutely solid mantle and a compressible liquid core with the simple fluid density distritution

$$
\rho(R)=\rho_{o}\left(1-\delta\left(R / R_{n}\right)^{2}\right)
$$

where $R_{n}$ is the core radius. Geometry of the equal-value density surfaces may be obtained from Clairaut's equation. Let the Brunt-Väisälä frequency equal zero. Then the fluid oscillations are described by the equations

$$
\begin{aligned}
& \Delta \psi-\frac{4 \omega^{2}}{\sigma^{2}} \frac{\partial^{2} \psi}{\partial z^{2}}=-\left(\sigma^{2}-4 \omega^{2}\right) \frac{P_{1}}{\rho \alpha^{2}}-\frac{\sigma^{2}-4 \omega^{2}}{i \sigma} v \frac{\nabla \rho}{\rho} \\
& \Delta V_{1}=4 \pi G \alpha^{-2}\left(\psi-V_{1}+V_{t}\right) \quad \psi=P_{1} / \rho+V_{1}+V_{t}
\end{aligned}
$$

where $\omega$ is the angular velocity, $\sigma$ the frequency, $\alpha$ the speed of sound, and $v, P_{1}, V_{1}$ are perturbations of velocity, pressure and gravitational potential, respectively; $V_{t}$ is the tide-generating potential. 
The first term in the right hand of Eq. (3) is negligible. Thus, only Eq. (3) needs to be solved. Let the solution be represented by expansion in characteristic functions of the Poincare operator:

$$
\begin{aligned}
& \psi=\left(a_{o}(x z-i y z)+\sum_{l} \sum_{k} \sum_{m} a^{m}{ }_{l k} \Psi^{m} l k\right) \exp (i \sigma t) \\
& \left(\Delta-\frac{\partial^{2}}{\partial z^{2}}\right) \Psi^{m} l k=\lambda^{m} l k \Psi^{m} l k
\end{aligned}
$$

Substitution of Eq. (5) into Eq. (3) gives a system of equations for $a^{m}{ }_{k \cdot}$. The non-diagonal elements of the matrix of this system are small in comparison with the diagonal ones. This makes it possible to truncate the expansion Eq. (5) in order to obtain an approximate solution. The first 36 characteristic numbers of the Poincaré operator for one of the frequencies are given in Table 1.

Table 1. Characteristic numbers of Poincare operator $(m=1)$

\begin{tabular}{lllrrrr}
\hline$l \backslash k$ & 1 & 2 & 3 & 4 & 5 & 6 \\
1 & -16.1103 & 0.1355 & 76.6711 & 235.8353 & 489.7864 & 843.0952 \\
2 & -58.0358 & -46.4465 & 8.3694 & 83.8804 & 197.9489 & 366.1997 \\
3 & -133.1034 & -126.2940 & -60.1717 & 15.9224 & 116.7159 & 243.3884 \\
4 & -245.1963 & -242.0729 & -155.8808 & -80.9797 & 23.6751 & 152.8642 \\
5 & -396.6794 & -395.5154 & -277.3416 & -208.0382 & -101.7706 & 31.6236 \\
6 & -588.3208 & -587.9475 & -425.0769 & -364.3752 & -258.0745 & -122.5184 \\
\hline
\end{tabular}

Nutation amplitudes for different maximum numbers of characteristic functions in Eq. (5) are given in table 2. For comparison, there are shown nutation amplitudes for the absolutely solid and the Poincaré models.

\begin{tabular}{|c|c|c|c|c|c|c|}
\hline & $\begin{array}{l}\text { Solid } \\
\text { model }\end{array}$ & $\begin{array}{l}\text { Poincare } \\
\text { model }\end{array}$ & $\begin{array}{l}\text { Compressib } \\
1=1, \mathrm{k}=2\end{array}$ & $\begin{array}{l}\text { e core model } \\
N=2\end{array}$ & $N=4$ & $N=6$ \\
\hline-6800 & 8051.05 & 7999.60 & 7992.19 & 7992.32 & 7992.42 & 7992.43 \\
\hline-365.3 & 24.91 & -38.633 & -28.757 & -28.905 & -29.028 & -29.042 \\
\hline-182.5 & 22.60 & 28.197 & 29.242 & 29.223 & 29.208 & 29.205 \\
\hline
\end{tabular}

Table 2. Nutation amplitudes $[\mathrm{N}=\max \{l, k\}]$

A good convergence of the proposed method makes it possible to use it while calculating the Earth nutation amplitudes and free nuclear oscillations. The method may be extended for the case of nonzero Brunt-Väisälä frequency. Extension of the method for the case of a solid inner core will be the subject of future investigations. Acknowledgements: The author is grateful to Prof. S.M. Molodenskii and to E.V. Andrjievskii, I.V. Kurushkin, V.R. Leschuk, and V.V. Zuikin.

\section{References}

1. Smith, M.L. 1974, "The scalar equations of infinitesimal elastic-gravitational motion for a rotating slightly-elliptical Earth", Geophys. J. Royal Astron. Soc. 37, 491-526.

2.. Crossly, D.J., Rochester, M.G. 1980, "Simple core undertones”, Geophys. J. Royal Astron. Soc. 60, 129-161.

3. Wahr, J.M. 1981, The forced nutation of an elliptical, rotating elastic and oceanless Earth, Geophys. J. Royal Astron. Soc. 64, 705-727. 